\title{
Nondense Subsets of Varieties in a Power of an Elliptic Curve
}

\section{Evelina Viada}

Département de Mathématiques, Université de Fribourg Suisse, Pérolles, Chemin du Musée 23, CH-1700 Fribourg, Switzerland

Correspondence to be sent to: viada@math.ethz.ch

Let $E$ be an elliptic curve without complex multiplication (CM) defined over $\overline{\mathbb{Q}}$. We show that on a transverse $d$-dimensional variety $V \subset E^{g}$, the set of algebraic points of bounded height, which are close to the union of all algebraic subgroups of $E^{g}$ of codimension $d+1$ translated by points in a subgroup $\Gamma$ of $E^{g}$ of finite rank, is Zariski nondense in $V$. The notion of close is defined using a height function. If $\Gamma=0$, it is sufficient to assume that $V$ is weak-transverse. This result is optimal with respect to the codimension of the algebraic subgroups. The method is based on an essentially optimal effective version of the Bogomolov Conjecture. Such an effective result is proven for subvarieties of $E^{g}$. If we assume that the sets have bounded height, then we can prove that they are not Zariski dense. A conjecture, known in some special cases, claims that the sets in question have bounded height. We prove here a new case. In conclusion, our results prove a generalized case of a conjecture by Zilber and by Pink in $E^{g}$.

\section{Introduction}

In this article all algebraic varieties are defined over $\overline{\mathbb{Q}}$ and we consider only algebraic points. Denote by $A$ an abelian variety of dimension $g$. Consider a proper irreducible algebraic subvariety $V$ of $A$ of dimension $d$. We say that:

- $\quad V$ is transverse, if $V$ is not contained in any translate of a proper algebraic subgroup of $A$. 
- $\quad V$ is weak-transverse, if $V$ is not contained in any proper algebraic subgroup of $A$.

Given an integer $r$ with $1 \leq r \leq g$ and a subset $F$ of $A$, we define the set

$$
S_{r}(V, F)=V \cap \bigcup_{\operatorname{cod} B \geq r}(B+F),
$$

where $B$ varies over all abelian subvarieties of $A$ of codimension at least $r$ and

$$
B+F=\{b+f: b \in B, f \in F\}
$$

Note that

$$
S_{r+1}(V, F) \subset S_{r}(V, F) .
$$

We denote the set $S_{r}\left(V, A_{\text {Tor }}\right)$ simply by $S_{r}(V)$, where $A_{\text {Tor }}$ is the torsion of $A$. For convenience, for $r>g$ we define $S_{r}(V, F)=\emptyset$ and for $V^{e}$ a subset of $V$ we define

$$
S_{r}\left(V^{e}, F\right)=V^{e} \cap S_{r}(V, F) .
$$

We ask for which sets $F$ and integers $r$ the set $S_{r}(V, F)$ has bounded height or is Zariski nondense in $V$.

Depending on the choice of $F$, the set $S_{g}(V, F)$ appears in the literature in the context of the Mordell-Lang, of the Manin-Mumford and of the Bogomolov Conjectures. More recently Bombieri, Masser, and Zannier [2] proved that for a transverse curve in a torus, the set $S_{2}(C)$ is finite. They investigated intersections with the union of all algebraic subgroups of a given codimension. This opens a vast number of conjectures for subvarieties of semi-abelian varieties.

In this article, we consider a variety in a power of an elliptic curve. In the first part of this work, we study the nondensity of $S_{d+1}(V, \cdot)$; the last part is dedicated to its height. Let $E$ be an elliptic curve without complex multiplication (CM). Consider on $E^{g}$ the line bundle $\mathcal{L}$ given as tensor product of the pull backs via the natural projections of a symmetric ample line bundle on $E$. We fix on $E^{g}$ a semi-norm $\|\cdot\|$ induced by the Néron-Tate height on $E$. For $\varepsilon \geq 0$, we denote

$$
\mathcal{O}_{\varepsilon}=\left\{\xi \in E^{g}:\|\xi\| \leq \varepsilon\right\}
$$

We denote by $\Gamma$ a subgroup of finite rank in $E^{g}$. We define $\Gamma_{\varepsilon}=\Gamma+\mathcal{O}_{\varepsilon}$. 
Let $V$ an irreducible algebraic subvariety of $E^{g}$ of dimension $d$. For a non-negative real $K$, we define

$$
V_{K}=V \cap \mathcal{O}_{K}
$$

Our main result is:

Theorem 1.1. For every $K \geq 0$ there exists an effective $\varepsilon>0$ such that:

(i) If $V$ is weak-transverse, $S_{d+1}\left(V_{K}, \mathcal{O}_{\varepsilon}\right)$ is Zariski nondense in $V$.

(ii) If $V$ is transverse, $S_{d+1}\left(V_{K}, \Gamma_{\varepsilon}\right)$ is Zariski nondense in $V$.

Because of the different hypotheses on the variety and the different sets in the thesis, there are no evident implications between the statements (i) and (ii).

Let us say at once that the theorem is expected to hold for $V$ instead of $V_{K}$. This is immediately implied by the following conjecture. Indeed $S_{d+1}(V, \cdot) \subset S_{d+1}\left(V^{u}, \cdot\right) \cup\left(V \backslash V^{u}\right)$. The conjecture gives $S_{d+1}\left(V^{u}, \cdot\right) \subset S_{d+1}\left(V_{K}, \cdot\right)$ for some non effective $K>0$.

Conjecture 1.2. There exist $\varepsilon>0$ and a nonempty Zariski open subset $V^{u}$ of $V$ such that:

(i) If $V$ is weak-transverse, $S_{d+1}\left(V^{u}, \mathcal{O}_{\varepsilon}\right)$ has bounded height.

(ii) If $V$ is transverse, $S_{d+1}\left(V^{u}, \Gamma_{\varepsilon}\right)$ has bounded height.

The method known to show that the height is bounded relies on a Vojta inequality, unless $\Gamma$ is trivial. This method gives optimal results for curves, while for varieties a hypothesis stronger than transversality is needed. Let $V \subset E^{g}$ be a variety of dimension $d$ such that

$$
\operatorname{dim}(V+B)=\min (d+\operatorname{dim} B, g)
$$

for all abelian subvarieties $B$ of $E^{g}$. In this article, we extend the proof of Rémond of Conjecture 1.2(ii) for $V$ satisfying condition (1). In Theorem 6.5, we prove Conjecture 1.2(i) for $V \times p$ where $p \in E^{s}$ is a point not lying in any proper algebraic subgroup of $E^{s}$. In Section 2, we clarify that, up to an isogeny of $E^{n}$, a weak-transverse variety in $E^{n}$ has the shape $V \times p$ for $V$ transverse in some $E^{g}$ and $p$ a point in $E^{n-g}$ not lying in any proper algebraic subgroup of $E^{n-g}$. We can then conclude:

Theorem 1.3. For $V$ satisfying condition (1) and $p \in E^{s}$ a point not lying in any proper algebraic subgroup of $E^{s}$, there exists $\varepsilon>0$ such that: 
(i) The set $S_{d+1}\left(V \times p, \mathcal{O}_{\varepsilon}\right)$ is Zariski nondense in $V \times p$.

(ii) The set $S_{d+1}\left(V, \Gamma_{\varepsilon}\right)$ is Zariski nondense in $V$.

For the codimension of the subgroups equal to $g$, statements (i) and (ii) are cases of the Bogomolov Conjecture and the Mordell-Lang plus Bogomolov Conjecture, respectively. Let us emphasise that our theorem neither gives a new proof of the Bogomolov Conjecture (as we make use of such a result), nor we get a new proof of the Mordell-Lang Conjecture (as we use a more general Vojta inequality). On the contrary we give a new proof of the Mordell-Lang plus Bogomolov Theorem (Poonen [7]), under the assumption (1). In addition, part (ii) of Theorem 1.3 proves a case of a conjecture by Zilber and Pink extended by the Bogomolov Conjecture.

In [14], we proved our main result for a curve in $E^{g}$. A naive extension of the method in [14], would imply a weak form of Theorem 1.1, where the codimension of the algebraic subgroups shall be at least $2 d$ instead of $d+1$. Here, we improve the method used in [14] obtaining the optimal $d+1$. In the first instance we show that Theorem 1.1 (i) and (ii) are equivalent, then we prove Theorem 1.1(ii).

Theorem 1.4. Given $K \geq 0$ and a positive integer $r$, the following statements are equivalent:

(i) For $V$ weak-transverse, there exists $\varepsilon>0$ such that $S_{r}\left(V_{K}, \mathcal{O}_{\varepsilon}\right)$ is Zariski nondense in $V$.

(ii) For $V$ transverse, there exists $\varepsilon>0$ such that $S_{r}\left(V_{K}, \Gamma_{\varepsilon}\right)$ is Zariski nondense in $V$.

We shall then prove Theorem 1.1, part (ii). Like for curves, the strategy of the proof is based on two steps. A union of infinitely many sets is Zariski nondense if:

(1) the union can be taken over finitely many sets,

(2) all sets in the union are Zariski nondense.

Part (1) is a typical problem of Diophantine approximation; we approximate an algebraic subgroup with a subgroup of bounded degree (see Proposition 3.3).

The second step (2) is a problem of height theory and its proof relies on an essentially optimal lower bound for the normalized height of a transverse subvariety in $E^{g}$, Theorem 1.5 below. This part is delicate. The dimension of the variety intervenes heavily on the estimates we provide. A fundamental idea is to reduce the problem to the study of varieties with finite stabilizer (see Section 4). 
We define $\mu(V)$ as the supremum of the reals $\epsilon(V)$ such that $S_{g}\left(V, \mathcal{O}_{\epsilon(V)}\right)=V \cap \mathcal{O}_{\epsilon(V)}$ is Zariski nondense in $V$. Work by Ullmo [12] and Zhang [15] proves the Bogomolov Conjecture. This shows that $\mu(V)>0$, for $V$ transverse. A first effective lower bound for $\mu(V)$ is provided by David and Philippon [3] Theorem 1.2. The type of bounds we need are an elliptic analogue of Amoroso and David [1] Theorem 1.4. Such a result is proven by Galateau in his $\mathrm{PhD}$ thesis for $d \geq g-2$, and in a preprint [4] for varieties in a product of elliptic curves with or without CM (he gives estimates the essential minimum of $V$ which is the square of $\mu(V))$.

Theorem 1.5 (Bogomolov-type bound, Galateau [4]). Let $V$ be a transverse subvariety of $E^{g}$ of codimension cod $V$. For $\eta>0$, there exists a positive effective constant $c\left(E^{g}, \eta\right)$ depending on the ambient variety and $\eta$, such that for

$$
\epsilon(V, \eta)=\frac{c\left(E^{g}, \eta\right)}{\left(\operatorname{deg}_{\mathcal{L}} V\right)^{\frac{1}{2 \operatorname{cod} V}+\eta}}
$$

the set

$$
V \cap \mathcal{O}_{\epsilon(V, \eta)}
$$

is Zariski nondense in $V$.

The bound $\epsilon(V, \eta)$ depends on the invariants of the ambient variety and on the degree of $V$. The quasi-optimal dependence on the degree of $V$ and the nondependence on the field of definition and height of $V$ are of crucial importance for our application.

The Zariski nondensity for transverse varieties has often been investigated with the method introduced by Bombieri, Masser, and Zannier in [2]. To show the nondensity property they use an essentially optimal Generalized Lehmer Conjecture. In [13] we applied their method to a transverse curve, $\Gamma=0$ and $\varepsilon=0$. In [8] Rémond and the author extended the method to transverse curves, $\varepsilon=0$ and any $\Gamma$ of finite rank. In [9-11] Rémond generalized it to varieties satisfying a geometric property stronger than transversality.

The main advantage of using a Bogomolov-instead of a Lehmer-type bound is that an essentially optimal generalized Lehmer conjecture has been proven for CM abelian varieties while it is not likely to be proven in a near future for non-CM abelian varieties. On the contrary, the Bogomolov-type bound has been proven at least for some non-CM abelian varieties. In addition, our method gives the nondensity for a neighborhood of positive radius $\varepsilon$. At present it is not known how to obtain results of this kind in abelian varieties using a Lehmer-type bound. 
The Zariski nondensity for a transverse subvariety in a torus and $\Gamma=0$ has been studied independently by Habegger [5]. He uses the Bogomolov-type bound proven by Amoroso and David [1] and proves that for a transverse variety $V$ in $\mathbb{G}_{m}^{n}$, there exists $\varepsilon>0$ such that the set $S_{2 d}\left(V, \mathcal{O}_{\varepsilon}\right)$ is Zariski nondense.

In the next section, we fix the notation and recall the results we need from [14]. In Section 3, we present the four main steps of the proof of Theorem 1.1. Section 4 is the core of this article: we prove the nondensity of the intersections. In Section 5, we conclude the proof of the main theorem. In the final section we prove that sometimes the height is bounded.

\section{Preliminaries}

In the following, we aim to be as transparent as possible, polishing statements from technicality. Therefore, we present the proofs for a power of an elliptic curve $E$ without CM. Then End(E) is identified with $\mathbb{Z}$. Proofs for a subvariety in a product of arbitrary elliptic curves are slightly more technical.

\subsection{Small points}

On $E$, we fix a symmetric very ample line bundle $\mathcal{L}_{0}$. On $E^{g}$, we consider the bundle $\mathcal{L}$, which is the tensor product of the pull-backs of $\mathcal{L}_{0}$ via the natural projections on the factors. Degrees are computed with respect to the polarization $\mathcal{L}$. Usually $E^{g}$ is endowed with the $\mathcal{L}$-canonical Néron-Tate height $h^{\prime}$. Though, we prefer to define on $E^{g}$ the height of the maximum

$$
h\left(x_{1}, \ldots, x_{g}\right)=\max _{i}\left(h\left(x_{i}\right)\right),
$$

where $h\left(x_{i}\right)$ on $E$ is given by the $\mathcal{L}_{0}$-canonical Néron-Tate height. Note that $h(x) \leq h^{\prime}(x) \leq$ $g h(x)$. Hence, the two norms induced by $h$ and $h^{\prime}$ are equivalent. We denote by $\|\cdot\|$ the seminorm induced by $h$ on $E^{g}$.

For $\varepsilon \geq 0$, we denote

$$
\mathcal{O}_{\varepsilon}=\left\{\xi \in E^{g}:\|\xi\| \leq \varepsilon\right\}
$$

\subsection{Morphisms and their height}

We denote by $M_{r, g}(\mathbb{Z})$ the module of $r \times g$ matrices with entries in $\mathbb{Z}$. For $F=\left(f_{i j}\right) \in M_{r, g}(\mathbb{Z})$, we define the height of $F$ as the maximum of the absolute value of its entries

$$
H(F)=\max _{i j}\left|f_{i j}\right|
$$


A morphism $\phi: E^{g} \rightarrow E^{r}$ is identified with an integral matrix. Let $a \in \mathbb{Z}$, we denote by $[a]$ the multiplication by $a$.

Note that, the set of morphisms of height less than a constant is a finite set.

\subsection{Algebraic subgroups}

Let $B$ be an algebraic subgroup of $E^{g}$ of codimension $r$. Then $B \subset \operatorname{ker} \phi_{B}$ for a surjective morphism $\phi_{B}: E^{g} \rightarrow E^{r}$. Conversely, we denote by $B_{\phi}$ the kernel of a surjective morphism $\phi: E^{g} \rightarrow E^{r}$. Then $B_{\phi}$ is an algebraic subgroup of $E^{g}$ of codimension $r$. Note that $r$ is the rank of $\phi$. An easy observation (see, for instance, [13], p. 61, line 3) gives that each of the $r$ equations defining $B_{\phi}$ has degree at most $H(\phi)^{2}$, up to a multiplicative constant depending on $\operatorname{deg} E$ and $g$. This directly implies:

Lemma 2.1. Let $\phi: E^{g} \rightarrow E^{r}$ be a surjective morphism. Then

$$
\operatorname{deg} B_{\phi} \leq c_{0} H(\phi)^{2 r}
$$

where $c_{0}$ is a constant depending on $\operatorname{deg} E$ and $g$.

\subsection{Subgroups}

Let $\Gamma$ be a subgroup of $E^{g}$ of finite rank $s$. Then $\Gamma$ is a $\mathbb{Z}$-module of rank $s$. We call a maximal free set of $\Gamma$ a set of $s$ linearly independent elements of $\Gamma$; in other words, a basis of $\Gamma \otimes_{\mathbb{Z}} \mathbb{Q}$. If $\Gamma$ is a free module, we call integral generators a set of $s$ generators of $\Gamma$.

The division group $\Gamma_{0}$ of the coordinates group of the points of $\Gamma$, in short of $\Gamma$, is a subgroup of $E$ defined as

$$
\Gamma_{0}=\left\{y \in E \text { such that } N_{y} \in \pi(\Gamma) \text { for } N \in \mathbb{Z}^{*} \text { and } \pi: E^{g} \rightarrow E\right\}
$$

Note that, $\Gamma_{0}^{g}=\Gamma_{0} \times \cdots \times \Gamma_{0}$ contains $\Gamma$ and it is a module of finite rank. This shows that, to prove nondensity statements for $\Gamma$ it is enough to prove them for $\Gamma_{0}^{g}$.

Definition 2.2. We say that a point $p=\left(p_{1}, \ldots, p_{n}\right) \in E^{n}$ has rank $s$ if its coordinates group $\left\langle p_{1}, \ldots, p_{n}\right\rangle$ has rank $s$. We define $\Gamma_{p}$ to be the division group of $\left\langle p_{1}, \ldots, p_{n}\right\rangle$.

Given a point $p \in E^{s}$ of rank $s$, we associate to $p$ a positive real $\varepsilon_{0}(p)$. This value will be used several times in the following. 
Proposition 2.3 ([14] Proposition 3.3 with $\tau=1, \operatorname{End}(\mathrm{E})=\mathbb{Z}, c_{0}(p)=c_{2}(p, 1)$ and $\varepsilon_{0}(p)=$ $\left.\varepsilon_{0}(p, 1)\right)$. Let $p_{1}, \ldots, p_{s}$ be linearly independent points of $E$ and $p=\left(p_{1}, \ldots, p_{s}\right)$. Then, there exist positive reals $c_{0}(p)$ and $\varepsilon_{0}(p)$ such that

$$
c_{0}(p) \sum_{i}\left|b_{i}\right|^{2}\left\|p_{i}\right\|^{2} \leq\left\|\sum_{i} b_{i}\left(p_{i}-\xi_{i}\right)-b \xi\right\|^{2}
$$

for all $b_{1}, \ldots, b_{s}, b \in \mathbb{Z}$ with $|b| \leq \max _{i}\left|b_{i}\right|$ and for all $\xi_{1}, \ldots, \xi_{s}, \xi \in E$ with $\left\|\xi_{i}\right\|,\|\xi\| \leq \varepsilon_{0}(p)$.

\subsection{From transverse to weak-transverse}

Let $V$ be transverse in $E^{g}$ and let $\Gamma$ be a subgroup of $E^{g}$ of finite rank. Let $\Gamma_{0}$ be the division group of $\Gamma$ and let $s$ be its rank. If $s=0$ we define $V^{\prime}=V$. If $s>0$, we denote by $\gamma_{1}, \ldots, \gamma_{s}$ a maximal free set of $\Gamma_{0}$ and

$$
\gamma=\left(\gamma_{1}, \ldots, \gamma_{s}\right)
$$

We define

$$
V^{\prime}=V \times \gamma
$$

Since $V$ is transverse and $\gamma$ has rank $s$, then $V^{\prime}$ is weak-transverse in $E^{g+s}$.

\subsection{From weak-transverse to transverse}

Let $V^{\prime}$ be weak-transverse in $E^{n}$. If $V^{\prime}$ is transverse then we define $V=V^{\prime}$ and $\Gamma=0$. If $V^{\prime}$ is not transverse, let $H_{0}$ be the abelian subvariety of smallest dimension $g$ such that $V^{\prime} \subset H_{0}+p^{\perp}$ for $p^{\perp} \in H_{0}^{\perp}$ and $H_{0}^{\perp}$ the orthogonal complement of $H_{0}$ of dimension $s=n-g$. Then $E^{n}$ is isogenous to $H_{0} \times H_{0}^{\perp}$. Furthermore, $H_{0}$ is isogenous to $E^{g}$ and $H_{0}^{\perp}$ is isogenous to $E^{s}$. Let $j_{0}, j_{1}$ and $j_{2}$ be such isogenies. We fix the isogeny

$$
j=\left(j_{1} \times j_{2}\right) \circ j_{0}: E^{n} \rightarrow H_{0} \times H_{0}^{\perp} \rightarrow E^{g} \times E^{s},
$$

which sends $H_{0}$ to $E^{g} \times 0$ and $H_{0}^{\perp}$ to $0 \times E^{s}$ and $j\left(p^{\perp}\right)=\left(0, \ldots, 0, p_{1}, \ldots, p_{s}\right)$. Since $V^{\prime}$ is weak-transverse and defined over $\overline{\mathbb{Q}}, p=\left(p_{1}, \ldots, p_{s}\right)$ has rank $s$ and is defined over $\overline{\mathbb{Q}}$. 
We consider the natural projection on the first $g$ coordinates:

$$
\begin{gathered}
\pi: E^{g} \times E^{S} \rightarrow E^{g} \\
j\left(V^{\prime}\right) \rightarrow \pi\left(j\left(V^{\prime}\right)\right) .
\end{gathered}
$$

We define

$$
V=\pi\left(j\left(V^{\prime}\right)\right)
$$

and

$$
\Gamma=\Gamma_{p}^{g} .
$$

Since $H_{0}$ has minimal dimension, the variety $V$ is transverse in $E^{g}$ and $\Gamma$ has rank gs. Finally,

$$
j\left(V^{\prime}\right)=V \times p .
$$

We remark that we have defined a bijection $\left(V, \Gamma_{0}^{g}\right) \rightarrow V^{\prime}$, which is exactly what interest us.

\subsection{Weak-transverse up to an isogeny}

Statements on boundedness of heights and nondensity of sets are invariant under an isogeny of the ambient variety. Namely, given an isogeny $j$ of $E^{g}$, Theorem 1.1 and Conjecture 1.2 hold for a variety if and only if they hold for its image via $j$. Thus, the previous discussion shows that without loss of generality, we can assume that a weaktransverse variety $V^{\prime}$ in $E^{n}$ is of the form

$$
V^{\prime}=V \times p,
$$

where

$$
\begin{aligned}
\text { (i) } & V \text { is transverse in } E^{g}, \\
\text { (ii) } & p=\left(p_{1}, \ldots, p_{s}\right) \text { is a point in } E^{s} \text { of rank } s, \\
\text { (iii) } & n=g+s .
\end{aligned}
$$

In short we will say that $V \times p$ is a weak-transverse variety in $E^{g+s}$, to say that $V$ is transverse in $E^{g}$ and $p \in E^{s}$ has rank $s$. This simplifies the setting for weak-transverse varieties. 


\subsection{Gauss-reduced morphisms}

The matrices in $M_{r \times g}(\mathbb{Z})$ of the form

$$
\phi=\left(a I_{r} \mid L\right)=\left(\begin{array}{cccccc}
a & \ldots & 0 & a_{1, r+1} & \ldots & a_{1, g} \\
\vdots & \vdots & \vdots & & \vdots \\
0 & \ldots & a & a_{r, r+1} & \ldots & a_{r, g}
\end{array}\right),
$$

with $H(\phi)=a$, will play a key role in this work. If $r=g$, simply forget $L$. The following definition of Gauss-reduced is slightly more general than the one given in [14]; namely we omit here the assumption that the entries of the matrix have no common factors. This is a marginal simplification, overseen in that article.

Definition 2.4 (Gauss-reduced morphisms). Given positive integers $g, r$, we say that a morphism $\phi: E^{g} \rightarrow E^{r}$ is Gauss-reduced if:

(i) There esists $a \in \mathbb{N}^{*}$ such that $a I_{r}$ is a submatrix of $\phi$, with $I_{r}$ the r-identity matrix,

(ii) $H(\phi)=a$.

A morphisms $\phi^{\prime}$, given by a reordering of the rows of a morphism $\phi$, has the same kernel as $\phi$. Saying that $a I_{r}$ is a submatrix of $\phi$ fixes one permutation of the rows of $\phi$.

A reordering of the columns corresponds, instead, to a permutation of the coordinates. Statements will be proven for Gauss-reduced morphisms of the form $\phi=\left(a I_{r} \mid L\right)$. For each other reordering of the columns, the proofs are analogous. Since there are finitely many permutations of $g$ columns, the nondensity statements will follow.

There are a few easy facts that one shall keep in mind. Let $\psi: E^{g} \rightarrow E^{r}$ be a morphism and $\phi: E^{g} \rightarrow E^{r}$ be a Gauss-reduced morphism, then

(i) For $x \in E^{g}$,

$$
\|\psi(x)\| \leq g H(\psi)\|x\|
$$

and

$$
\|\phi(x)\| \leq(g-r+1) a\|x\| .
$$

(ii) For $x \in E^{r} \times\{0\}^{g-r}$,

$$
\phi(x)=[a] x .
$$


The following lemma shows that every abelian subvariety of codimension $r$ is contained in the kernel of a Gauss-reduced morphism of rank $r$.

Lemma 2.5 ([14] Lemma 4.4(ii) with $\operatorname{End}(\mathrm{E})=\mathbb{Z}$ ). Let $\psi: E^{g} \rightarrow E^{r}$ be a morphism of rank $r$. Then, there exists a Gauss-reduced morphism $\phi: E^{g} \rightarrow E^{r}$ such that

$$
B_{\psi} \subset B_{\phi}+\left(E_{\text {Tor }}^{r} \times\{0\}^{g-r}\right) .
$$

Taking intersections with $V_{K}$, the previous lemma translates immediately as:

Lemma 2.6. For any reals $K \geq 0, \varepsilon \geq 1$ and integer $r \geq 1$, it holds

$$
S_{r}\left(V_{K},\left(\Gamma_{0}^{g}\right)_{\varepsilon}\right)=\bigcup_{\substack{\phi: E^{g} \rightarrow E^{r} \\ \text { Gauss-reduced }}} V_{K} \cap\left(B_{\phi}+\left(\Gamma_{0}^{g}\right)_{\varepsilon}\right) .
$$

\subsection{Quasi-special and Special morphisms}

Special morphisms play a key role in the study of weak-transverse varieties. A Special morphism $\tilde{\phi}$ is Gauss-reduced. In addition, the multiplication by $H(\tilde{\phi})$ acts on some of the first $g$-coordinates.

Definition 2.7 (Quasi-special and Special morphisms). Given positive integers $g, s, r$, a morphism $\tilde{\phi}: E^{g+s} \rightarrow E^{r}$ is Quasi-special if there exist a Gauss-reduced morphism $\phi: E^{g} \rightarrow E^{r}$ and a morphism $\phi^{\prime}: E^{s} \rightarrow E^{r}$ such that

(i) $\tilde{\phi}=\left(\phi \mid \phi^{\prime}\right)$.

The morphism $\tilde{\phi}: E^{g+s} \rightarrow E^{r}$ is Special if it satisfies the further condition

(ii) $H(\tilde{\phi})=H(\phi)$.

Note that, for $g=2$ and $r=s=1$, the morphism $(0,0,1)$ is Gauss-reduced, but not Special. While $(1,0,2)$ is Quasi-special but not Special. In addition, for $g=r=2, s=1$, $\phi=\left(\left.I_{2}\right|_{3} ^{2}\right)$ is Quasi-special but not Gauss-reduced.

We want to show that if a point of large rank is in the kernel of a morphism then it is in the kernel of a Quasi-special morphism.

Lemma 2.8. Let $V$ be an algebraic subvariety of $E^{g}$. Let $p=\left(p_{1}, \ldots, p_{s}\right)$ be a point in $E^{s}$ of rank $s$. There exists $\varepsilon_{0}(p)>0$ depending on $p$ such that for all $\varepsilon \leq \varepsilon_{0}(p)$, for any subset 
$V^{e}$ of $V$ and positive integer $r$ it holds

$$
S_{r}\left(V^{e} \times p, \mathcal{O}_{\varepsilon}\right) \subset \bigcup_{\begin{array}{c}
\tilde{\phi}: E^{g+s} \rightarrow E^{r} \\
\text { Quasi-special }
\end{array}}\left(V^{e} \times p\right) \cap\left(B_{\tilde{\phi}}+\mathcal{O}_{\varepsilon}\right)
$$

Proof. The proof is the analog of Lemma 6.2 of [14], where we shall read $V^{e}$ for $C$.

\section{The Proof of Theorem 1.1: The Four Main Steps}

In the following, we present the four main steps for the proof of Theorem 1.1.

(0) We prove Theorem 1.4, which claims that Theorem 1.1 (i) and (ii) are equivalent. We then shall prove Theorem 1.1(ii).

(1) In Proposition 3.2, we get rid of $\Gamma$ by considering instead of $V$ the weaktransverse variety $V \times \gamma$, where $\gamma$ is a maximal free set of $\Gamma_{0}$. The key point is that for $V \times \gamma$ we consider

$$
\bigcup_{\substack{\tilde{\phi}: E^{g+s} \rightarrow E^{d+1} \\ \text { Special }}}\left(V_{K} \times \gamma\right) \cap\left(B_{\tilde{\phi}}+\mathcal{O}_{\delta}\right)
$$

where the union ranges only over Special morphisms (and not over all Gaussreduced morphisms).

(2) In Proposition 3.3, we show that the above union is contained in the union of finitely many sets of the kind

$$
\left(V_{K} \times \gamma\right) \cap\left(B_{\tilde{\phi}}+\mathcal{O}_{\delta^{\prime} / H(\tilde{\phi})^{1+\frac{1}{2 n}}}\right)
$$

Important is that the radius of the neighborhood of these finitely many sets is inversally proportional to the height of the morphism (and it is not a constant $\delta$ like in the union in step (1)).

(3) In Proposition 4.4, we show that if the stabilizer of $V$ is finite, then there exists $\varepsilon>0$ such that, for all Special morphisms $\tilde{\phi}$ of rank at least $d+1$, the set

$$
\left(V_{K} \times \gamma\right) \cap\left(B_{\tilde{\phi}}+\mathcal{O}_{\delta / H(\tilde{\phi})}\right)
$$

is Zariski nondense in $V \times \gamma$. 
The statements (0), (1), and (2) are an immediate generalization of [14] Theorem 1.3, Proposition 10.2, and Proposition A, respectively. Part (3) is the most delicate and it is presented in Section 4, below. It is the counterpart to [14] Proposition B. In order to gain advantage from Theorem 1.5, we need to require that the stabilizer of the variety is finite. In view of Lemma 5.1, this assumption will not be restrictive.

Part (0). Theorem 1.4 is an immediate consequence of

Theorem 3.1. Let $V$ be an irreducible algebraic subvariety of $E^{g}$. Then, for $\varepsilon \geq 0$ and $r$ a positive integer:

(i) The map $x \rightarrow(x, \gamma)$ defines an injection

$$
S_{r}\left(V, \Gamma_{\varepsilon}\right) \hookrightarrow S_{r}\left(V \times \gamma, \mathcal{O}_{\varepsilon}\right) .
$$

Recall that $\gamma$ is a maximal free set of the division group $\Gamma_{0}$ of $\Gamma$.

Let $p \in E^{s}$ be a point of rank $s$ and $K \geq 0$. Then, there exists $\varepsilon_{0}(p)>0$ such that:

(ii) For $\varepsilon \leq \varepsilon_{0}(p)$, the map $(x, p) \rightarrow x$ defines an injection

$$
S_{r}\left(V_{K} \times p, \mathcal{O}_{\varepsilon}\right) \hookrightarrow S_{r}\left(V_{K^{\prime}}\left(\Gamma_{p}^{g}\right)_{\varepsilon K^{\prime}}\right),
$$

where $K^{\prime}=(g+s) \max \left(1, \frac{g(K+\varepsilon)}{c(p)}\right)$ and $c(p)$ is a positive constant depending on $p$. Recall that $\Gamma_{p}$ is the division group of the coordinates of $p$.

Proof. The proof is the analog of the proof of [14] Theorem 9.1, where we shall read $V$ for $C, K$ for $K_{3}, \varepsilon_{0}(p)$ for $\varepsilon_{p}$ and $K^{\prime}$ for $K_{4}$. Note that the inequality $\|x\| \leq K$ is insured by considering just points in $V_{K}$ (unlike in [14] where $\|x\| \leq K_{3}$ is due to the hypothesis $r \geq 2$ and $\left.\varepsilon \leq \varepsilon_{3}\right)$.

Part (1). Given a subgroup $\Gamma$ and a real $K,[14]$ Lemma 3.4 (with End(E) $=\mathbb{Z})$ proves that there exists a maximal free set $\gamma_{1}, \ldots, \gamma_{s}$ of the division group $\Gamma_{0}$ such that

$$
\begin{aligned}
\left\|\gamma_{i}\right\| & \geq 3 g K, \\
\left\|\sum_{i} b_{i} \gamma_{i}\right\|^{2} & \geq \frac{1}{9} \sum_{i}\left|b_{i}\right|^{2}\left\|\gamma_{i}\right\|^{2}
\end{aligned}
$$

for $b_{1}, \ldots, b_{s} \in \mathbb{Z}$. We define

$$
\gamma=\left(\gamma_{1}, \ldots, \gamma_{s}\right)
$$

with $\gamma_{i}$ satisfying the above conditions. 
Proposition 3.2. Let $V$ be an irreducible algebraic subvariety of $E^{g}$. For $r$ a positive integer, $K \geq 0$ and $\varepsilon \leq \frac{K}{g}$, the map $x \rightarrow(x, \gamma)$ defines an injection

$$
\bigcup_{\begin{array}{c}
\phi: E^{g} \rightarrow E^{r} \\
\text { Gauss-reduced }
\end{array}} V_{K} \cap\left(B_{\phi}+\left(\Gamma_{0}^{g}\right)_{\varepsilon}\right) \hookrightarrow \bigcup_{\begin{array}{c}
\tilde{\phi}=\left(\phi \mid \phi^{\prime}\right) \\
\text { Special }
\end{array}}\left(V_{K} \times \gamma\right) \cap\left(B_{\tilde{\phi}}+\mathcal{O}_{\varepsilon}\right) .
$$

Proof. The proof is the analog of Proposition 10.2 of [14], where one shall read $K$ for $K_{1}$, $V$ for $C, V_{K}$ for $C(\overline{\mathbb{Q}})$. Note that, here, the estimate $\|x\| \leq K$ is ensured by the assumption that we consider points in $V_{K}$ (unlike in [14], where it is due to the assumptions $r \geq 2$ and $\left.\varepsilon \leq \varepsilon_{1}\right)$.

Part (2).

Proposition 3.3. Let $V$ be an irreducible algebraic subvariety of $E^{g}$. Let $p=\left(p_{1}, \ldots, p_{s}\right) \in$ $E^{s}$ be a point of rank $s$. Then, for $r$ a positive integer, $K \geq 0$ and $\varepsilon>0$,

$$
\bigcup_{\substack{\tilde{\phi}: E^{g+s} \rightarrow E^{r} \\ \text { Special }}}\left(V_{K} \times p\right) \cap\left(B_{\tilde{\phi}}+\mathcal{O}_{\varepsilon / M^{1+\frac{1}{2 n}}}\right) \subset \bigcup_{\substack{\tilde{\psi}: E^{g+s} \rightarrow E^{r} \\ \text { Special, } H(\tilde{\psi}) \leq M}}\left(V_{K} \times p\right) \cap\left(B_{\tilde{\psi}}+\mathcal{O}_{(g+s+1) \varepsilon / H(\tilde{\psi})^{1+\frac{1}{2 n}}}\right),
$$

where $M=\max \left(2,\left\lceil\frac{K+\|p\|}{\varepsilon}\right\rceil^{2}\right)^{n}$ and $n=r(g+s)-r^{2}+1$.

Proof. The proof is the analog of the proof of Proposition A, part (ii) of [14], where one shall read $V_{K}$ instead of $C(\overline{\mathbb{Q}}), p$ for $\gamma, K$ for $K_{2}$, and $M$ for $M^{\prime}$. And where the estimate $\|x\| \leq K$ is ensured by the assumption that we consider points in $V_{K}$ (and not as in [14], where it is due to the hypothesis $r \geq 2$ and $\varepsilon \leq \varepsilon_{2}$ ).

Note that in the last row of the proof in [14], we estimate $g-r+1+s+1$ with $g+s$, because $r \geq 2$. Here we instead estimate $g-r+1+s+1$ with $g+s+1$, because $r \geq 1$.

\section{The Proof of Theorem 1.1: Part (3)}

Recall that $\mu(V)$ is the supremum of the reals $\epsilon(V)$ such that $V \cap \mathcal{O}_{\epsilon(V)}$ is Zariski nondense in $V$. The essential minimum of $V$ is the square of $\mu(V)$. Using Theorem 1.5, we produce a sharp lower bound for the essential minimum of the image of a variety under a Gaussreduced morphism. Unlike for curves, the stabilizer of the variety will play quite an important role. In this section, we will often assume that $V$ has finite stabilizer. In 
Lemma 5.1, we will see that such an assumption is not restrictive for the proof of our main theorem.

\subsection{The estimate for the essential minimum}

Consider a Gauss-reduced morphism $\phi$ of codimension $r=d+1$ :

$$
\phi=\left(\begin{array}{c}
\varphi_{1} \\
\vdots \\
\varphi_{r}
\end{array}\right)=\left(\begin{array}{cccc}
a & \ldots & 0 & L_{1} \\
\vdots & \ddots & \vdots & \vdots \\
0 & \ldots & a & L_{r}
\end{array}\right)
$$

where $L_{i} \in \mathbb{Z}^{g-r}$. We denote $\bar{x}=\left(x_{r+1}, \ldots, x_{g}\right)$.

We define the isogenies:

$$
\begin{aligned}
F & : E^{g} \rightarrow E^{g} \\
& \left(x_{1}, \ldots, x_{g}\right) \rightarrow\left(x_{1}, \ldots, x_{r}, a x_{r+1}, \ldots, a x_{g}\right) \\
L: & E^{g} \rightarrow E^{g} \\
& \left(x_{1}, \ldots, x_{g}\right) \rightarrow\left(x_{1}+L_{1}(\bar{x}), \ldots, x_{r}+L_{r}(\bar{x}), x_{r+1}, \ldots, x_{g}\right) ; \\
\Phi: & E^{g} \rightarrow E^{g} \\
& \left(x_{1}, \ldots, x_{g}\right) \rightarrow\left(\varphi_{1}(x), \ldots, \varphi_{r}(x), x_{r+1}, \ldots, x_{g}\right) .
\end{aligned}
$$

Definition 4.1 Helping-variety. We define the variety

$$
W=L F^{-1}(V)
$$

Then

$$
\Phi(V)=[a] W .
$$

We now estimate degrees.

Proposition 4.2. There exist positive constants $c_{1}$ and $c_{2}$ depending on $g$ and $\operatorname{deg} E$ such that:

(i) The degree of $\phi(V)$ is bounded by $c_{1} a^{2 d} \operatorname{deg} V$. 
Suppose further that $V$ has finite stabilizer. Then,

(ii) The degree of $W$ is bounded by $c_{2} a^{2(g-r)}|\operatorname{Stab} V| \operatorname{deg} V$.

Proof. For simplicity we indicate by « an inequality up to a multiplicative constant depending on $g$ and $\operatorname{deg} E$.

Let $X$ be an irreducible algebraic subvariety of $E^{g}$.

First we estimate the degree of the image of $X$ under an isogeny $\psi: E^{g} \rightarrow E^{g}$. According to the chosen polarization

$$
\operatorname{deg} \psi(X)=\sum_{I} E_{i_{1}} \cdots E_{i_{d}} \cdot \psi(X)
$$

where $I=\left(i_{1}, \ldots, i_{d}\right)$ ranges over the possible combinations of $d$ elements in the set $\{1, \ldots, g\}$ and $E_{i_{j}}$ is the coordinate subgroup given by $x_{i_{j}}=0$. Then

$$
\operatorname{deg} \psi(X) \ll \max _{I}\left(E_{i_{1}} \cdots E_{i_{d}} \cdot \psi(X)\right) .
$$

Let us estimate the intersection numbers on the right. By definition,

$$
E_{i_{1}} \cdots E_{i_{d}} \cdot \psi(X)=B_{\psi_{I}} \cdot X
$$

where the rows of $\psi_{I}$ are the $i_{1}, \ldots, i_{d}$ rows of $\psi$. Note that rk $\psi_{I}=d$ and $H\left(\psi_{I}\right) \leq H(\psi)$. Bezout's Theorem and Lemma 2.1 (applied with $\phi=\psi_{I}$ and $r=d$ ) give

$$
B_{\psi_{I}} \cdot X \leq \operatorname{deg} B_{\psi_{I}} \operatorname{deg} X \ll H\left(\psi_{I}\right)^{2 d} \operatorname{deg} X \ll H(\psi)^{2 d} \operatorname{deg} X .
$$

We conclude

$$
\operatorname{deg} \psi(X) \ll H(\psi)^{2 d} \operatorname{deg} X
$$

For $\psi=\Phi$, we deduce

$$
\operatorname{deg} \Phi(V) \ll H(\Phi)^{2 d} \operatorname{deg} V=a^{2 d} \operatorname{deg} V
$$

(i) In the chosen polarization, forgetting coordinates makes degrees decrease.

Note that $\phi(V)=\pi \Phi(V)$, where $\pi$ is the projection on the first $r$ coordinates. By (5), we conclude that

$$
\operatorname{deg} \phi(V) \leq \operatorname{deg} \Phi(V) \ll a^{2 d} \operatorname{deg} V
$$

(ii) In [6] Lemma 6, part (i), Hindry proves: 
For any positive integer $b$,

$$
\operatorname{deg}[b] X=\frac{b^{2 d}}{\left|\operatorname{Stab} X \cap E^{g}[b]\right|} \operatorname{deg} X,
$$

where $|\cdot|$ means the cardinality of a set and $E^{g}[b]$ is the kernel of the multiplication $[b]$. Recall that $\Phi(V)=[a] W$. We deduce that

$$
\operatorname{deg} \Phi(V)=\operatorname{deg}[a] W=\frac{a^{2 d}}{\left|\operatorname{Stab} W \cap E^{g}[a]\right|} \operatorname{deg} W
$$

Thus

$$
\operatorname{deg} W=\frac{\left|\operatorname{Stab} W \cap E^{g}[a]\right|}{a^{2 d}} \operatorname{deg} \Phi(V)
$$

By relation (5), we deduce

$$
\operatorname{deg} W \ll\left|\operatorname{Stab} W \cap E^{g}[a]\right| \operatorname{deg} V .
$$

We now estimate the cardinality of the stabilizer of $W$. Since $W=L F^{-1} V$, we get

$$
\text { Stab } W=L F^{-1} \text { Stab } V
$$

More precisely, if $x \in$ Stab $W$ then $x+W \subset W$. Recall that $L$ is an isomorphism. Applying $F L^{-1}$ on both sides, we obtain $F L^{-1} X+V \subset V$. Thus $F L^{-1} X \in$ Stab $V$ and $x \in L F^{-1}$ Stab $V$. On the other hand, suppose that $x \in L F^{-1}$ Stab $V$. Then $F L^{-1} X+V \subset V$. Considering the pre-image, $x+\operatorname{ker}\left(F L^{-1}\right)+W \subset W$. But, by definition, $W$ is $\operatorname{ker}\left(F L^{-1}\right)$ invariant, so $x+$ $W \subset W$ and $x \in$ Stab $W$.

By assumption, the stabilizer of $V$ is finite. In addition, $L$ is an isomorphism. So

$$
\mid \text { Stab } W|=| \operatorname{ker} F|| \text { Stab } V\left|=a^{2(g-r)}\right| \text { Stab } V \mid \text {. }
$$

In view of (6), we conclude that

$$
\operatorname{deg} W \ll|\operatorname{Stab} W| \operatorname{deg} V \ll a^{2(g-r)} \mid \text { Stab } V \mid \operatorname{deg} V
$$

The following proposition is a lower bound for the essential minimum of the image of a variety under Gauss-reduced morphisms. It reveals the dependence on the 
height of the morphism. While the first bound is an immediate application of Theorem 1.5 and Proposition 4.2, the second estimate is subtle.

Proposition 4.3. Let $\phi$ be a Gauss-reduced morphism of rank $d+1$ with $a=H(\phi)$. Then, for any point $y \in E^{g}$ and any $\eta>0$,

$$
\mu(\phi(V+y))>\epsilon_{1}(V, \eta) \frac{1}{a^{d+2 d \eta}},
$$

where $\epsilon_{1}(V, \eta)$ is an effective positive constant depending on $V, E, g$, and $\eta$. Suppose further that $V$ has finite stabilizer. Let $\Phi$ be the isogeny defined in (4). Then

(ii)

$$
\mu(\Phi(V+y))>\epsilon_{2}(V, \eta) a^{\frac{1}{g-d}-2(g-d-1) \eta},
$$

where $\epsilon_{2}(V, \eta)$ is an effective positive constant depending on $V, E, g$, and $\eta$.

Proof. Let us recall the Bogomolov-type bound given in Theorem 1.5; for a transverse irreducible variety $X$ in $E^{g}$ and any $\eta>0$,

$$
\mu(X)>\epsilon(X, \eta)=\frac{C\left(E^{g}, \eta\right)}{\operatorname{deg} X^{\frac{1}{2 \operatorname{cod} X}+\eta}} .
$$

(i) Let $q=\phi(y)$. Then $\phi(V+y)=\phi(V)+q$. Since $V$ is irreducible, transverse, and defined over $\overline{\mathbb{Q}}, \phi(V)+q$ is so as well.

Observe that $\phi(V) \subset E^{d+1}$ has dimension at least 1 (because $V$ is transverse) and at most $d$ (because dimension can just decrease under morphisms). Furthermore, dimensions are preserved by translations.

The bound (7) for $\phi(V)+q$ and $g=d+1$ gives

$$
\begin{aligned}
\mu(\phi(V+y)) & =\mu(\phi(V)+q) \\
& >\epsilon(\phi(V)+q, \eta)=\frac{C\left(E^{d+1}, \eta\right)}{(\operatorname{deg}(\phi(V)+q))^{\frac{1}{2 \operatorname{cod} \phi(V)}+\eta}} \\
& \geq \frac{C\left(E^{d+1}, \eta\right)}{(\operatorname{deg}(\phi(V)+q))^{\frac{1}{2}+\eta}} .
\end{aligned}
$$


Degrees are preserved by translations, hence Proposition 4.2(i) implies

$$
\operatorname{deg}(\phi(V)+q)=\operatorname{deg} \phi(V) \leq c_{1} a^{2 d} \operatorname{deg} V .
$$

If follows

$$
\epsilon(\phi(V)+q, \eta) \geq \frac{C\left(E^{d+1}, \eta\right)}{\left(C_{1} a^{2 d} \operatorname{deg} V\right)^{\frac{1}{2}+\eta}} .
$$

Define

$$
\epsilon_{1}(V, \eta)=\frac{C\left(E^{d+1}, \eta\right)}{\left(C_{1} \operatorname{deg} V\right)^{\frac{1}{2}+\eta}}
$$

Then

$$
\mu(\phi(V+y))>\frac{\epsilon_{1}(V, \eta)}{a^{d+2 d \eta}} .
$$

(ii) Let $q \in E^{g}$ be a point such that $[a] q=\Phi(y)$. Let $W_{0}$ be an irreducible component of $W=L F^{-1}(V)$. Then

$$
\Phi(V+y)=[a]\left(W_{0}+q\right) .
$$

Therefore

$$
\mu(\Phi(V+y))=a \mu\left(W_{0}+q\right)
$$

We now estimate $\mu\left(W_{0}+q\right)$ via the bound (7). The variety $W_{0}+q \subset E^{g}$ is irreducible by definition. Since $V$ is transverse and defined over $\overline{\mathbb{Q}}, W_{0}+q$ is so as well. Furthermore, isogenies and translations preserve dimensions. Thus $\operatorname{dim}\left(W_{0}+q\right)=\operatorname{dim} V=$ $d$. Then,

$$
\mu\left(W_{0}+q\right)>\epsilon\left(W_{0}+q, \eta\right)=\frac{c\left(E^{g}, \eta\right)}{\operatorname{deg}\left(W_{0}+q\right)^{\frac{1}{2(g-d)}+\eta}} .
$$

Since $W_{0}$ is an irreducible component of $W, \operatorname{deg} W_{0} \leq \operatorname{deg} W$. Furthermore, translations by a point preserve degrees. Thus, Proposition 4.2(ii) with $r=d+1$ gives

$$
\operatorname{deg}\left(W_{0}+q\right) \leq \operatorname{deg} W \leq c_{2} a^{2(g-d-1)}|\operatorname{Stab} V| \operatorname{deg} V .
$$


Therefore

$$
\mu\left(W_{0}+q\right)>\frac{C\left(E^{g}, \eta\right)}{\left(c_{2}|\operatorname{Stab} V| \operatorname{deg} V\right)^{\frac{1}{2(g-d)}+\eta}}\left(a^{2(g-d-1)}\right)^{-\frac{1}{2(g-d)}-\eta} .
$$

Define

$$
\epsilon_{2}(V, \eta)=\frac{c\left(E^{g}, \eta\right)}{\left(c_{2}|\operatorname{Stab} V| \operatorname{deg} V\right)^{\frac{1}{2(g-d)}+\eta}} .
$$

So

$$
\mu\left(W_{0}+q\right)>\epsilon_{2}(V, \eta) a^{-1+\frac{1}{g-d}-2(g-d-1) \eta} .
$$

Replace in (8), to obtain

$$
\mu(\Phi(V+y))>\epsilon_{2}(V, \eta) a^{\frac{1}{g-d}-2(g-d-1) \eta}
$$

\subsection{The nondensity of the intersections}

We come to the main proposition of this section: each set in the union is Zariski nondense. The proof of (i) of case (1) is delicate. In general $\mu(\pi(V)) \leq \mu(V)$ for $\pi$ a projection on some factors. We shall rather find a kind of reverse inequality. On a set of bounded height this will be possible.

Proposition 4.4. Suppose that $V \subset E^{g}$ has finite stabilizer. Then, for every $K \geq 0$, there exists an effective $\varepsilon_{1}>0$ such that:

(i) For $\varepsilon \leq \varepsilon_{1}$, for all Gauss-reduced morphisms $\phi: E^{g} \rightarrow E^{d+1}$ and for all $y \in$ $E^{d+1} \times\{0\}^{g-d-1}$, the set

$$
\left(V_{K}+y\right) \cap\left(B_{\phi}+\mathcal{O}_{\varepsilon / H(\phi)}\right)
$$

is Zariski nondense in $V$.

(ii) Let $s$ be a positive integer. For $\varepsilon \leq \frac{\varepsilon_{1}}{g+s}$, for all Special morphisms $\tilde{\phi}=\left(\phi \mid \phi^{\prime}\right)$ : $E^{g+s} \rightarrow E^{d+1}$ and for all points $p \in E^{s}$, the set

$$
\left(V_{K} \times p\right) \cap\left(B_{\tilde{\phi}}+\mathcal{O}_{\varepsilon / H(\phi)}\right)
$$

is Zariski nondense in $V \times p$. 
Proof. Define

$$
\begin{aligned}
\eta & =\frac{1}{2 d}, \\
m & =\left(\frac{K}{\epsilon_{2}(V, \eta)}\right)^{\frac{g-d}{1-2(g-d-1)(g-d) \eta}}, \\
\varepsilon_{1} & =\min \left(\frac{K}{g}, \frac{\epsilon_{1}(V, \eta)}{g m^{d+1}}\right),
\end{aligned}
$$

where $\epsilon_{1}(V, \eta)$ and $\epsilon_{2}(V, \eta)$ are as in Proposition 4.3.

\section{Part (i).}

Let $a=H(\phi)$. We distinguish two cases:

(1) $a \geq m$,
(2) $a \leq m$.

Case (1): If $a \geq m,\left(V_{K}+y\right) \cap\left(B_{\phi}+\mathcal{O}_{\varepsilon / H(\phi)}\right)$ is nondense in $V$.

Let $x+y \in\left(V_{K}+y\right) \cap\left(B_{\phi}+\mathcal{O}_{\varepsilon / a}\right)$, where

$$
y=\left(y_{1}, \ldots, y_{d+1}, 0, \ldots, 0\right) \in E^{d+1} \times\{0\}^{g-d-1}
$$

Then

$$
\phi(x+y)=\phi(\xi)
$$

for $\|\xi\| \leq \varepsilon / a$.

Let $\Phi=\phi \times i d_{E^{g-d-1}}$ as in (4). Then

$$
\begin{aligned}
\Phi(x+y) & =\left(\phi(x+y), x_{d+2}, \ldots, x_{g}\right) \\
& =\left(\phi(\xi), x_{d+2}, \ldots, x_{g}\right) .
\end{aligned}
$$

Therefore

$$
\|\Phi(x+y)\|=\left\|\left(\phi(\xi), x_{d+2}, \ldots, x_{g}\right)\right\| \leq \max (\|\phi(\xi)\|,\|x\|) .
$$

Since $\|\xi\| \leq \frac{\varepsilon}{a}$ and $\varepsilon \leq \frac{K}{g}$

$$
\|\phi(\xi)\| \leq g \varepsilon \leq K
$$


Also, $\|x\| \leq K$, because $x \in V_{K}$. Thus,

$$
\|\Phi(x+y)\| \leq K
$$

We work under the hypothesis $a \geq m \geq\left(\frac{K}{\epsilon_{2}(V, \eta)}\right)^{\frac{g-d}{1-2(g-d-1)(g-d) \eta}}$; then

$$
K \leq \epsilon_{2}(V, \eta) a^{\frac{1}{g-d}-2(g-d-1) \eta} .
$$

In Proposition 4.3(ii), we have proven

$$
\epsilon_{2}(V, \eta) a^{\frac{1}{g-d}-2(g-d-1) \eta}<\mu(\Phi(V+y))
$$

So

$$
\|\Phi(x+y)\| \leq K<\mu(\Phi(V+y)) .
$$

We deduce that $\Phi(x+y)$ belongs to the Zariski nondense set

$$
Z_{1}=\Phi(V+y) \cap \mathcal{O}_{K}
$$

The restriction morphism $\Phi_{\mid V+y}: V+y \rightarrow \Phi(V+y)$ is generically finite, because $\Phi$ is an isogeny. Then $x+y$ belongs to the Zariski nondense set $\Phi_{\mid V+Y}^{-1}\left(Z_{1}\right)$.

We can conclude that, for every $\phi$ Gauss-reduced of rank $d+1$ with $H(\phi) \geq m$, the set

$$
\left(V_{K}+y\right) \cap\left(B_{\phi}+\mathcal{O}_{\varepsilon / H(\phi)}\right)
$$

is Zariski nondense.

Case (2): If $a \leq m,\left(V_{K}+y\right) \cap\left(B_{\phi}+\mathcal{O}_{\varepsilon / H(\phi)}\right)$ is nondense in $V$.

Let $x+y \in\left(V_{K}+y\right) \cap\left(B_{\phi}+\mathcal{O}_{\varepsilon / a}\right)$, where $y \in E^{d+1} \times\{0\}^{g-d-1}$. Then

$$
\phi(x+y)=\phi(\xi)
$$


for $\|\xi\| \leq \varepsilon / a$. However, we have chosen $\varepsilon \leq \epsilon_{1}(V, \eta) / g m^{d+1}$. Hence

$$
\|\phi(x+y)\|=\|\phi(\xi)\| \leq g \varepsilon \leq \frac{\epsilon_{1}(V, \eta)}{m^{d+1}} .
$$

We are working under the hypothesis $a \leq m$. Moreover, $\eta=\frac{1}{2 d}$. Then

$$
a^{d+2 d \eta} \leq m^{d+1}
$$

Thus

$$
\|\phi(x+y)\| \leq \frac{\epsilon_{1}(V, \eta)}{m^{d+1}} \leq \frac{\epsilon_{1}(V, \eta)}{a^{d+2 d \eta}}
$$

In Proposition 4.3(i), we have proven

$$
\frac{\epsilon_{1}(V, \eta)}{a^{d+2 d \eta}}<\mu(\phi(V+y))
$$

We deduce that $\phi(x+y)$ belongs to the Zariski nondense set

$$
Z_{2}=\phi(V+y) \cap \mathcal{O}_{\epsilon_{1}(V, \eta) / m^{d+1}}
$$

Since $V$ is transverse, the dimension of $\phi(V+y)$ is at least 1 . Consider the restriction morphism $\phi_{\mid V+y}: V+y \rightarrow \phi(V+y)$. Then $x+y$ belongs to the Zariski nondense set $\phi_{\mid V+y}^{-1}\left(Z_{2}\right)$. We conclude that, for all $\phi$ Gauss-reduced of rank $d+1$ with $H(\phi) \leq m$, the set

$$
\left(V_{K}+y\right) \cap\left(B_{\phi}+\mathcal{O}_{\varepsilon / H(\phi)}\right)
$$

is Zariski nondense.

Cases (1) and (2) prove part (i).

Part (ii). We are going to show that, for every $\tilde{\phi}=\left(\phi \mid \phi^{\prime}\right)$ Special of rank $d+1$ (note that $\phi$ is Gauss-reduced of rank $d+1$ ), there exists $y \in E^{d+1} \times\{0\}^{g-d-1}$ such that the map $(x, p) \rightarrow x+y$ defines an injection

$$
\left(V_{K} \times p\right) \cap\left(B_{\tilde{\phi}}+\mathcal{O}_{\varepsilon / H(\phi)}\right) \hookrightarrow\left(V_{K}+y\right) \cap\left(B_{\phi}+\mathcal{O}_{(g+s) \varepsilon / H(\phi)}\right)
$$


We then apply part (i) of this proposition to $\phi$ and $y$; since $(g+s) \varepsilon \leq \varepsilon_{1}$,

$$
\left(V_{K}+y\right) \cap\left(B_{\phi}+\mathcal{O}_{(g+s) \varepsilon / H(\phi)}\right)
$$

is Zariski nondense in $V$. So for $\varepsilon \leq \frac{\varepsilon_{1}}{g+s}$, the set

$$
\left(V_{K} \times p\right) \cap\left(B_{\tilde{\phi}}+\mathcal{O}_{\varepsilon / H(\phi)}\right)
$$

is Zariski nondense in $V$.

Let us prove the inclusion (9). Let $\tilde{\phi}=\left(\phi \mid \phi^{\prime}\right)$ be Special of rank $d+1$. By definition of Special, $\phi=\left(a I_{d+1} \mid L\right)$ is Gauss-reduced of rank $d+1$.

Let $y^{\prime} \in E^{d+1}$ be a point such that

$$
[a] y^{\prime}=\phi^{\prime}(p) .
$$

Define

$$
y=\left(y^{\prime}, 0, \ldots, 0\right) \in E^{d+1} \times\{0\}^{g-d-1} .
$$

Then

$$
\phi(y)=[a] y^{\prime}=\phi^{\prime}(p)
$$

Let

$$
(x, p) \in\left(V_{K} \times p\right) \cap\left(B_{\tilde{\phi}}+\mathcal{O}_{\varepsilon / a}\right) .
$$

Then, there exists $\xi \in \mathcal{O}_{\varepsilon / a}$ such that

$$
\tilde{\phi}((x, p)+\xi)=0 .
$$

Equivalently,

$$
\phi(x)+\phi^{\prime}(p)+\tilde{\phi}(\xi)=0
$$

and

$$
\phi(x+y)+\tilde{\phi}(\xi)=0 .
$$

Let $\xi^{\prime \prime} \in E^{d+1}$ be a point such that

$$
[a] \xi^{\prime \prime}=\tilde{\phi}(\xi)
$$


We define $\xi^{\prime}=\left(\xi^{\prime \prime},\{0\}^{g-d-1}\right)$, then

$$
\phi\left(\xi^{\prime}\right)=[a] \xi^{\prime \prime}=\tilde{\phi}(\xi)
$$

and

$$
\phi\left(x+y+\xi^{\prime}\right)=0
$$

Since $\tilde{\phi}$ is Special, $H(\tilde{\phi})=a$. Further, $\|\xi\| \leq \frac{\varepsilon}{a}$. We deduce

$$
\left\|\xi^{\prime}\right\|=\left\|\xi^{\prime \prime}\right\|=\frac{\|\tilde{\phi}(\xi)\|}{a} \leq \frac{(g+s) \varepsilon}{a} .
$$

In conclusion,

$$
\phi\left(x+y+\xi^{\prime}\right)=0
$$

with $\left\|\xi^{\prime}\right\| \leq \frac{(g+s) \varepsilon}{a}$. Equivalently,

$$
(x+y) \in\left(V_{K}+y\right) \cap\left(B_{\phi}+\mathcal{O}_{(g+s) \varepsilon / H(\phi)}\right),
$$

where $y \in E^{d+1} \times\{0\}^{g-d-1}$ and $\phi$ is Gauss-reduced of rank $d+1$.

This proves relation (9) and concludes the proof.

\section{The Proof of Theorem 1.1: Conclusion}

\subsection{Reducing to a variety with finite stabilizer}

In the following lemma, we will show that to prove Theorem 1.1 it is sufficient to prove it for varieties with finite stabilizer. This innocent remark will allow us to use all results of Section 4.

Lemma 5.1. They hold:

(i) Let $X=X_{1} \times E^{d_{2}}$ be a subvariety of $E^{g}$ of dimension d. Then, for $r \geq d_{2}$,

$$
S_{r}(X, F) \hookrightarrow S_{r-d_{2}}\left(X_{1}, F^{\prime}\right) \times E^{d_{2}},
$$

where $F^{\prime}$ is the projection of $F$ on $E^{g-d_{2}}$. 
(ii) Let $V$ be a (weak)-transverse subvariety of $E^{g}$. Suppose that $\operatorname{dim}$ Stab $V=$ $d_{2} \geq 1$. Then, there exists an isogeny $j$ of $E^{g}$ such that

$$
j(V)=V_{1} \times E^{d_{2}}
$$

with $V_{1}$ (weak)-transverse in $E^{g-d_{2}}$ and Stab $V_{1}$ a finite group.

(iii) Theorem 1.1 holds if and only if it holds for varieties with finite stabilizer.

Proof. (i) Let $\left(x_{1}, x_{2}\right) \in S_{r}(X, F)$ with $x_{1} \in X_{1}$ and $x_{2} \in E^{d_{2}}$. Then, there exist $\phi: E^{g} \rightarrow E^{r}$ of rank $r$ and $\left(f_{1}, f_{2}\right) \in F$ such that

$$
\phi\left(\left(x_{1}, x_{2}\right)-\left(f_{1}, f_{2}\right)\right)=0 .
$$

Decompose $\phi=(\alpha \mid \beta)$ with $\alpha: E^{g-d_{2}} \rightarrow E^{r}$ and $\beta: E^{d_{2}} \rightarrow E^{r}$. Note that rk $\beta=r_{2} \leq d_{2}$ because of the number of columns. Then, the Gauss algorithm ensures the existence of an invertible matrix $\Delta \in \mathrm{GL}_{r}(\mathbb{Z})$ such that

$$
\Delta \phi=\left(\begin{array}{cc}
\phi_{1} & 0 \\
\star & \phi_{2}
\end{array}\right),
$$

where $\phi_{1}: E^{g-d_{2}} \rightarrow E^{r-r_{2}}$ and $\phi_{2}: E^{d_{2}} \rightarrow E^{r_{2}}$ of rank $r_{2}$.

Since $r=\operatorname{rk} \phi=\operatorname{rk} \phi_{1}+\operatorname{rk} \phi_{2}$, we deduce $\mathrm{rk} \phi_{1}=r-r_{2} \geq r-d_{2}$. Furthermore, relation (10) implies

$$
\phi_{1}\left(x_{1}-f_{1}\right)=0
$$

Thus $x_{1} \in S_{r-d_{2}}\left(X_{1}, F^{\prime}\right)$.

(ii) Let $\mathrm{Stab}^{0} V$ be the zero component of Stab $V$. Consider the projection

$$
\pi_{S}: E^{g} \rightarrow E^{g} / \operatorname{Stab}^{0} V
$$

Define $V_{1}^{\prime}=\pi_{S}(V)$. Then

$$
\operatorname{dim} V_{1}^{\prime}=\operatorname{dim}\left(V+\operatorname{Stab}^{0} V\right)-\operatorname{dim} \operatorname{Stab}^{0} V=d-d_{2}<g-d_{2}
$$

Since $V$ is (weak)-transverse and $\operatorname{dim} V_{1}^{\prime}<g-d_{2}$, then $V_{1}^{\prime}$ is (weak)-transverse in $E^{g} / \operatorname{Stab}^{0} V$. Let $\left(\mathrm{Stab}^{0} V\right)^{\perp}$ be the orthogonal complement of $\operatorname{Stab}^{0} V$ in $E^{g}$ and let 
$j_{0}: E^{g} / \operatorname{Stab}^{0} V \rightarrow\left(\operatorname{Stab}^{0} V\right)^{\perp}$ be an isogeny. Define the isogeny

$$
\begin{aligned}
j^{\prime}: E^{g} & \rightarrow\left(E^{g} / \operatorname{Stab}^{0} V\right) \times \operatorname{Stab}^{0} V, \\
x & \rightarrow\left(\pi_{S}(x), x-j_{0}\left(\pi_{S}(x)\right) .\right.
\end{aligned}
$$

Then

$$
j^{\prime}(V) \subset V_{1}^{\prime} \times \operatorname{Stab}^{0} V .
$$

Since these varieties have the same dimension and are irreducible,

$$
j^{\prime}(V)=V_{1}^{\prime} \times \operatorname{Stab}^{0} V .
$$

Let $i_{0}: E^{g} / \operatorname{Stab}^{0} V \rightarrow E^{g-d_{2}}$ and $i_{1}: \operatorname{Stab}^{0} V \rightarrow E^{d_{2}}$ be isogenies. Define $i=i_{0} \times i_{1}, j=i \circ j^{\prime}$ and $V_{1}=i\left(V_{1}^{\prime}\right)$. Then

$$
j(V)=V_{1} \times E^{d_{2}},
$$

with $V_{1}$ (weak)-transverse in $E^{g-d_{2}}$. Finally,

$$
\operatorname{Stab} V_{1}=i \circ \pi_{S}(\operatorname{Stab} V)
$$

is finite.

(iii) Suppose that $V$ is (weak)-transverse in $E^{g}$ and that $\operatorname{dim} \operatorname{Stab} V=d_{2}>0$, then, by part (ii), we can fix an isogeny $j$ such that $j(V)=V_{1} \times E^{d_{2}}$ with Stab $V_{1}$ a finite group and $V_{1}$ (weak)-transverse in $E^{g-d_{2}}$ of dimension $d_{1}=d-d_{2}$. Furthermore, by part (i) with $X=j(V), X_{1}=V_{1}, r=d+1$ and $F=\Gamma_{\varepsilon}$, we know that

$$
S_{d+1}\left(V, \Gamma_{\varepsilon}\right) \hookrightarrow S_{d_{1}+1}\left(V_{1}, \Gamma_{\varepsilon}^{\prime}\right) \times E^{d_{2}}
$$

So, if $S_{d_{1}+1}\left(V_{1}, \Gamma_{\varepsilon}^{\prime}\right)$ is Zariski nondense in $V_{1}$, also $S_{d+1}\left(V, \Gamma_{\varepsilon}\right)$ is Zariski nondense in $V$.

We can now conclude the proof of our main theorem. Let us recall that in view of Theorem 1.4, it is sufficient to prove part (ii). 
Proof of Theorem 1.1(ii). In view of Lemma 5.1 (iii), we can assume that Stab $V$ is finite. Recall that $r=d+1$, the rank of $\Gamma_{0}$ is $s$ and $n=(d+1)(g+s)-(d+1)^{2}+1$. Let $\gamma=\left(\gamma_{1}, \ldots, \gamma_{s}\right)$ be a point of rank $s$, such that $\gamma_{i}$ is a maximal free set of $\Gamma_{0}$ satisfying conditions (3).

\section{Choose}

(i) $\delta_{1}=\frac{1}{(g+s+1)} \min \left(\frac{\varepsilon_{1}}{g+s}, K\right)$, where $\varepsilon_{1}$ is as in Proposition 4.4,

(ii) $\delta=\delta_{1} M^{-1-\frac{1}{2 n}}$, where $M=\max \left(2,\left\lceil\frac{K+\|\gamma\|}{\delta_{1}}\right\rceil^{2}\right)^{n}$.

Since $\Gamma_{\delta} \subset\left(\Gamma_{0}^{g}\right)_{\delta}$, then

$$
S_{d+1}\left(V_{K}, \Gamma_{\delta}\right) \subset S_{d+1}\left(V_{K},\left(\Gamma_{0}^{g}\right)_{\delta}\right)
$$

Lemma 2.6, with $\varepsilon=\delta$ and $r=d+1$, shows that

$$
S_{d+1}\left(V_{K},\left(\Gamma_{0}^{g}\right)_{\delta}\right)=\bigcup_{\substack{\phi: E^{g} \rightarrow E^{d+1} \\ \text { Gauss-reduced }}} V_{K} \cap\left(B_{\phi}+\left(\Gamma_{0}^{g}\right)_{\delta}\right)
$$

Note that $\delta<\delta_{1} \leq \frac{K}{g}$. Then Proposition 3.2 with $\varepsilon=\delta$ implies

$$
\bigcup_{\begin{array}{c}
\phi: E^{g} \rightarrow E^{d+1} \\
\text { Gauss-reduced }
\end{array}} V_{K} \cap\left(B_{\phi}+\left(\Gamma_{0}^{g}\right)_{\delta}\right) \hookrightarrow \bigcup_{\begin{array}{c}
\tilde{\phi}=\left(\phi \mid \phi^{\prime}\right) \\
\text { Special }
\end{array}}\left(V_{K} \times \gamma\right) \cap\left(B_{\tilde{\phi}}+\mathcal{O}_{\delta}\right) .
$$

Note that $\delta_{1}>0$ and $\delta=\delta_{1} M^{-\left(1+\frac{1}{2 n}\right)}$. Then Proposition 3.3, with $\varepsilon=\delta_{1}, r=d+1$ and $p=\gamma$, shows that

$$
\bigcup_{\substack{\tilde{\phi}: E^{g+s} \rightarrow E^{d+1} \\ \text { Special }}}\left(V_{K} \times \gamma\right) \cap\left(B_{\tilde{\phi}}+\mathcal{O}_{\delta}\right)
$$

is a subset of

$$
Z=\bigcup_{\substack{\tilde{\phi}: E^{g+s} \rightarrow E^{d+1} \\ \text { Special, } H(\tilde{\phi}) \leq M}}\left(V_{K} \times \gamma\right) \cap\left(B_{\tilde{\phi}}+\mathcal{O}_{(g+s+1) \delta_{1} / H(\tilde{\phi})^{1+\frac{1}{2 n}}}\right)
$$

Observe that $Z$ is the union of finitely many sets, because $H(\tilde{\phi})$ is bounded by $M$. 
We have chosen $\delta_{1} \leq \varepsilon_{1} /(g+s+1)(g+s)$; moreover, Stab $V$ is finite. Then Proposition 4.4 (ii), with $\varepsilon=(g+s+1) \delta_{1} \leq \frac{\varepsilon_{1}}{g+s}$ and $p=\gamma$, implies that for all $\tilde{\phi}=\left(\phi \mid \phi^{\prime}\right)$ Special of rank $d+1$, the set

$$
\left(V_{K} \times \gamma\right) \cap\left(B_{\tilde{\phi}}+\mathcal{O}_{(g+s+1) \delta_{1} / H(\phi)}\right)
$$

is Zariski nondense in $V \times \gamma$. Note that $H(\phi) \leq H(\tilde{\phi})$, thus also the sets

$$
\left(V_{K} \times \gamma\right) \cap\left(B_{\tilde{\phi}}+\mathcal{O}_{(g+s+1) \delta_{1} / H(\tilde{\phi})^{1+\frac{1}{2 n}}}\right)
$$

are Zariski nondense. So $Z$ is Zariski nondense, because it is the union of finitely many Zariski nondense sets. We conclude that $S_{d+1}\left(V_{K}, \Gamma_{\delta}\right)$ is included in the Zariski nondense set $Z$.

Remark 5.2. In [14] we defined a different helping curve $W^{\prime}=A_{0}^{-1} W$ with $W$ as in Definition 4.1 and $A_{0}=\left(I_{2} \mid a_{0} I_{g-2}\right)$. This more complicated $W^{\prime}$ is needed because in [14] we produced a worse bound for the degree of $W^{\prime}$. Consequently, we proved a "weak" Proposition 4.4: we needed to assume that the neighborhoods have radius $\varepsilon / a_{0} a$. To compensate this loss, we needed the "strong" Proposition 3.3, where the radius is $\varepsilon / a_{0} a$. This was sufficient to prove our main theorem for curves. Such a trick is not sufficient to prove an optimal result for varieties.

In the present work, using the stabilizer, we produce a "good" bound for the degree of $W$, and we can prove the "strong" Proposition 4.4 for neighborhoods of radius $\varepsilon / a$. Then, to prove our main theorem in general, it is sufficient to use a "weak" Proposition 3.3 , where the radius of the neighborhoods is $\varepsilon / a$.

If we try to combine both "strong" statements, namely Proposition 3.3 (with $\left.\varepsilon / a_{0} a\right)$ and the "good" bound for the degree of $W$, we do not get any relevant improvement. Indeed, in the proof of Proposition 4.4, part (i), the inequality $\|x\| \leq K$ remains unchanged. The advantage would only be in respect of $\varepsilon$ in the statement of Proposition 4.4, where we could choose $\varepsilon \leq \epsilon_{1} m$.

\section{A Special Case of Conjecture 1.2}

The natural rising question is to investigate the height property for the codimension of the algebraic subgroups at least $d+1$. We expect that Conjecture 1.2 holds. The known results regarding this conjecture are based on a Vojta inequality, unless $\Gamma$ is trivial. 
Following Rémond's work, we prove here a new case of Conjecture 1.2. In this section, $E$ is a general elliptic curve (never mind whether CM or not). In view of Proposition 5.1 of Rémond [10], we give the following:

Definition 6.1. We say that a subset $V^{e}$ of $V$ satisfies a Vojta inequality if there exist real constants $c_{1}, c_{2}, c_{3}>0$ such that for $x_{1}, \ldots, x_{d+1} \in V^{e}$ with $\left\|x_{i}\right\| \geq c_{3}$ and for $\phi$ Gauss-reduced of rank $r \leq g$, there exists $s_{1}, \ldots, s_{d+1} \in \mathbb{N}^{*}$ with $s_{i} \geq c_{2} s_{i+1}$ such that

$$
\sum_{i=2}^{d+1}\left\|s_{i} \phi\left(x_{i}\right)-s_{1} \phi\left(x_{1}\right)\right\|^{2} \geq \frac{H(\phi)^{2}}{c_{1}} \sum_{i=1}^{d+1} s_{i}^{2}\left\|x_{i}\right\|^{2} .
$$

Note that a Gauss-reduced morphism is a normalized projector in the sense of [10]. Then, this definition tells us that if Proposition 5.1 of [10] holds for points in $V^{e}$ then $V^{e}$ satisfies a Vojta inequality.

Theorem 6.2 (Theorem 1.2 of Rémond [10]). If $V^{e} \subset V$ satisfies a Vojta inequality, then there exists $\varepsilon>0$ such that $S_{d+1}\left(V^{e}, \Gamma_{\varepsilon}\right)$ has bounded height.

Rémond also gives a definition of a candidate $V^{e}$ that satisfies a Vojta inequality and is possibly a nonempty open subset in $V$. In a recent article he shows:

Theorem 6.3 (Rémond [11]). Assume that $V \subset E^{g}$ satisfies condition (1). Then there exists a nonempty open subset $V^{u}$ of $V$ such that $V^{u}$ satisfies a Vojta inequality.

These two theorems imply:

Theorem 6.4. Conjecture 1.2(ii) holds for $V$ satisfying condition (1).

Here, we extend his theorem to the associated weak-transverse case.

Theorem 6.5. Conjecture 1.2(i) holds for $V \times p$, where $V$ satisfies condition (1) and $p$ is a point in $E^{s}$ not lying in any proper algebraic subgroup of $E^{s}$.

For $V$ transverse and $p \in E^{s}$ a point of rank $s$, we cannot embed the set $S_{r}(V \times$ $\left.p, \mathcal{O}_{\varepsilon}\right)$ in a set of the type $S_{r}\left(V, \Gamma_{\varepsilon^{\prime}}\right)$, unless we know a priori that the first set has bounded height. So, Theorem 6.2 is not enough to deduce a statement for $V \times p$.

However, we can embed $S_{r}\left(V \times p, \mathcal{O}_{\varepsilon}\right)$ in the union of two sets $S_{r}\left(V, \Gamma_{\varepsilon^{\prime}}\right) \cup(V \cap$ $\left.G_{p, \varepsilon, r}\right)$, where the set $G_{p, \varepsilon, r}$ is defined in the proof of Theorem 6.10 below. The same 
method can be used to show that, for $V^{e}$ satisfying a Vojta inequality, $V^{e} \cap G_{p, \varepsilon, r}$ has bounded height, exactly as we do for curves in Theorem 1.2 of [14].

Let us write the details.

Definition 6.6. Let $r, s$ positive integers and $\varepsilon>0$ a real. Let $p$ be a point in $E^{s}$. We define $G_{p}^{\varepsilon, r}$ as the set of points $\theta \in E^{r}$ for which there exist a matrix $A \in M_{r, s}(\operatorname{End}(\mathrm{E})$ ), an element $a \in \operatorname{End}(\mathrm{E})$ with $0<|a| \leq H(A)$, points $\xi \in E^{s}$ and $\zeta \in E^{r}$ of norm at most $\varepsilon$ such that

$$
[a] \theta=A(p+\xi)+[a] \zeta
$$

We identify $G_{p}^{\varepsilon, r}$ with the subset $G_{p}^{\varepsilon, r} \times\{0\}^{g-r}$ of $E^{g}$.

Lemma 6.7. Let $V$ be a subvariety of $E^{g}$. Let $V^{e}$ be a subset of $V$ and let $p \in E^{s}$ be a point. Then, for every $\varepsilon \geq 0$, the projection on the first $g$ coordinates

$$
\begin{gathered}
E^{g} \times E^{s} \rightarrow E^{g}, \\
(x, y) \rightarrow x,
\end{gathered}
$$

defines an injection

$$
S_{r}\left(V^{e} \times p, \mathcal{O}_{\varepsilon / 2 g s}\right) \hookrightarrow V^{e} \cap \bigcup_{\substack{\phi: E^{g} \rightarrow E^{r} \\ \text { Gauss-reduced }}}\left(B_{\phi}+\left(\Gamma_{p}^{g}\right)_{\varepsilon}\right) \cup\left(B_{\phi}+G_{p}^{\varepsilon, r}\right) .
$$

Proof. The proof is the analog of the proof of [14] Lemma 7.2, where we shall replace $C(\overline{\mathbb{Q}})$ by $V^{e}$, the codimension 2 by $r$ (as well as $E^{2}$ and $g-2$ by $E^{r}$ and $g-r$ ), the set $G_{p}^{\varepsilon}$ by $G_{p}^{\varepsilon, r}$. Also, we shall use Lemma 2.8 stated in this article, instead of Lemma 6.2 of [14] to which we refer there.

Lemma 6.8 Counterpart to Lemma 6.1 of [10]. For $\phi: E^{g} \rightarrow E^{r}$ Gauss-reduced of rank $r$, we have the following inclusion of sets:

$$
\left(B_{\phi}+G_{p}^{\varepsilon, r}\right) \subset\left\{P+\theta: P \in B_{\phi}, \theta \in G_{p}^{\varepsilon, r} \text { and } \max (\|\theta\|,\|P\|) \leq 2 g\|P+\theta\|\right\} .
$$

Proof. The proof is the analog of [14] Lemma 7.3, where one replaces $G_{p}^{\varepsilon}$ by $G_{p}^{\varepsilon, r}$ and 2 by $r$. 
Note that Lemma 6.2, part (1), of [10] is a statement on the morphisms; therefore it holds with no need of any remarks.

Lemma 6.9 Counterpart to Lemma 6.2, part (2), of [10]. Let $c_{1}$ be a given constant. Let $p \in E^{s}$ be a point of rank $s$. There exists $\varepsilon_{3}>0$ such that if $\varepsilon \leq \varepsilon_{3}$ then any sequence of elements in $G_{p}^{\varepsilon, r}$ admits a subsequence in which every two elements $\theta, \theta^{\prime}$ satisfy

$$
\left\|\frac{\theta}{\|\theta\|}-\frac{\theta^{\prime}}{\left\|\theta^{\prime}\right\|}\right\| \leq \frac{1}{16 g c_{1}} .
$$

Proof. The proof is the analog of [14] Lemma 7.4, where $A, A^{\prime} \in M_{r, s}(\operatorname{End}(\mathrm{E}))$ and $A=$ $\left(\begin{array}{c}A_{1} \\ \vdots \\ A_{r}\end{array}\right)$, with $A_{i} \in M_{1, s}(\operatorname{End}(\mathrm{E}))$.

We are ready to conclude.

Theorem 6.10. Let $p \in E^{s}$ be a point of rank $s$. Suppose that $V^{e} \subset V$ satisfies a Vojta inequality. Then, there exists $\varepsilon>0$ such that

$$
S_{d+1}\left(V^{e} \times p, \mathcal{O}_{\varepsilon}\right)
$$

has bounded height.

Proof. Define

$$
\Gamma_{\varepsilon, r}=\bigcup_{\substack{\phi: E^{g} \rightarrow E^{r} \\ \text { Gauss-reduced }}}\left(B_{\phi}+\left(\Gamma_{p}^{g}\right)_{\varepsilon}\right)
$$

and

$$
G_{p, \varepsilon, r}=\bigcup_{\begin{array}{c}
\phi: E^{g} \rightarrow E^{r} \\
\text { Gauss-reduced }
\end{array}}\left(B_{\phi}+G_{p}^{\varepsilon, r}\right)
$$

In view of Lemma 6.7, $S_{d+1}\left(V^{e} \times p, \mathcal{O}_{\varepsilon}\right) \hookrightarrow\left(V^{e} \cap \Gamma_{\varepsilon, d+1}\right) \cup\left(V^{e} \cap G_{p, \varepsilon, d+1}\right)$.

Theorem 6.2 shows that there exists $\varepsilon_{1}>0$ such that for $\varepsilon \leq \varepsilon_{1}, V^{e} \cap \Gamma_{\varepsilon, d+1}=$ $S_{d}\left(V^{e}, \Gamma_{\varepsilon}\right)$ has bounded height. 
It remains to show that there exists $\varepsilon_{2}>0$ such that for $\varepsilon \leq \varepsilon_{2}$, the set $V^{e} \cap G_{p, \varepsilon, d+1}$ has bounded height. The proof follows, step by step, the proof of Rémond [10], Theorem 1.2 , pp. 341-43, where one shall read $G_{p, \varepsilon, r}$ for $\Gamma_{\varepsilon, r}, \theta$ for $\gamma, V^{e}$ for $X(\overline{\mathbb{Q}}) \backslash Z_{X}^{(r)}$. Note that he writes $|\cdot|$ for the height norm, here we write $\|\cdot\|$. For the morphisms he uses a norm denoted by $\|\cdot\|$, here we denote the norm of a morphism by $H(\cdot)$. Lemmas 6.1 and 6.2 of [10] are replaced by our Lemmas 6.8 and 6.9. The Vojta inequality, Proposition 5.1 of [10], holds for the set $V^{e}$ by assumption.

Proof of Theorem 6.5. Thanks to Theorem 6.3, there exists a nonempty open subset $V^{u}$ of $V$ such that $V^{u}$ satisfies a Vojta inequality. Theorem 6.10 applied with $V^{e}=V^{u}$ implies that there exists $\varepsilon>0$ such that $S_{d+1}\left(V^{u} \times p, \mathcal{O}_{\varepsilon}\right)$ has bounded height.

In conclusion, Conjecture 1.2(i) and (ii) are not equivalent, but the same method can be applied to prove both cases.

\section{Acknowledgments}

I kindly thank the referee for his valuable suggestions. This work was supported by the Swiss National Science Foundation.

\section{References}

[1] Amoroso, F., and S. David. "Minoration de la hauteur normalisée dans un tore." Journal de l'Institut de Mathématiques de Jussieu 3, no. 2 (2003): 335-81.

[2] Bombieri, E., D. Masser, and U. Zannier. "Intersecting a curve with algebraic subgroups of multiplicative groups." International Mathematics Research Notices 20 (1999): 1119-40.

[3] David, S., and P. Philippon. "Minorations des hauteurs normalisées des sous-variétés de variétés abeliennes 2." Commentarii Mathematici Helvetici 4, no. 77 (2002): 639-700.

[4] Galateau, A. "Une Minorations du minimum essentiel sur les variétés abéliennes." (2008): preprint http://arxiv.org/PS_cache/arxiv/pdf/0807/0807.0171v1.pdf.

[5] Habegger, P. “A Bogomolov property for curves modulo algebraic subgroups." Bulletin de la Société Mathématique de France 1, no. 137 (2009): (forthcoming).

[6] Hindry, M. “ Autour d'une conjecture de Serge Lang." Inventiones Mathematicae 94 (1988): 575-603.

[7] Poonen, B. “Mordell-Lang plus Bogomolov." Inventiones Mathematicae 137 (1999): 413-25.

[8] Rémond, G., and E. Viada. "Problème de Mordell-Lang modulo certaines sous-variétés abéliennes." International Mathematics Research Notices 35 (2003): 1915-31.

[9] Rémond, G. "Intersection de sous-groups et de sous-variétés 1." Mathematische Annalen 333 (2005): 525-48. 
[10] Rémond, G. "Intersection de sous-groupes et de sous-variétés 2." Journal de l'Institut de Mathématiques de Jussieu 6 (2007): 317-48.

[11] Rémond, G. "Intersection de sous-groups et de sous-variétés 3." Commentarii Mathematici Helvetici (forthcoming).

[12] Ullmo, E. "Positivité et discrétion des points algébriques des courbes." Annals of Mathematics 1, no. 147 (1998): 167-79.

[13] Viada, E. "The intersection of a curve with algebraic subgroups in a product of elliptic curves." Annali della Scuola Normale Superiore di Pisa: Classe di Scienze 5, no. 2 (2003): 47-75.

[14] Viada, E. “ The intersection of a curve with a union of translated codimension-two subgroups in a power of an elliptic curve." Algebra and Number Theory 3, no. 2 (2008): 249-98.

[15] Zhang, S. "Equidistribution of small points on abelian varieties." Annals of Mathematics 1, no. 147 (1998): 159-65. 